\title{
Lifestyle Advice Provision to Teenage and Young Adult Cancer \\ Patients: The Perspective of Health Professionals in the UK
}

In preparation for: Supportive Care in Cancer

Pugh, Gemma. BSc, PhD Student ${ }^{1}$

Hough, Rachael. MD, Consultant Haematologist ${ }^{2}$

Gravestock, Helen. MA, Research and Policy Manager, CLIC Sargent ${ }^{3}$

Williams, Kate, BSc, MSc, PhD ${ }^{1}$

Fisher, Abigail. BSc, PhD, Lecturer in Physical Activity and Health ${ }^{1}$

${ }^{1}$ Health Behaviour Research Centre, University College London, 1-19 Torrington Place, London, WC1E 6BT

${ }^{2}$ University College Hospital's NHS Foundation Trust, 235 Euston Rd, London NW1 2BU

${ }^{3}$ CLIC Sargent, No.1 Farriers Yard, Assembly London, 77-85 Fulham Palace Road, London, W6 8JA, United Kingdom

Corresponding Author: abigail.fisher@ucl.ac.uk

\author{
Author Emails \\ abigail.fisher@ucl.ac.uk \\ k.williams.11@ucl.ac.uk \\ helen.gravestock@clicsargent.org.uk \\ rachael.hough@uclh.nhs.uk \\ gemma.pugh.14@ucl.ac.uk
}




\section{Abstract}

Purpose: Health professionals are an important source of information for teenage and young adult (TYA) cancer patients. However, little is known about health professionals' provision of lifestyle advice to young people with cancer who are in their care.

Methods: An online survey was distributed to health professionals within the UK who identified themselves as working with TYA cancer patients. Health professionals awareness of lifestyle guidance, provision of lifestyle advice to young people, and views on lifestyle information format and delivery were explored.

Results: Ninety-five health professionals (44\% nurses; $28 \%$ allied health professionals; $17 \%$ physicians) completed the survey. The majority (72\%) of respondents were aware of some lifestyle guidance for cancer patients. However, less than half of TYA health professionals (46\%) were able to successfully recall the source of the guidelines and less than a third reported proving specific advice to the majority of their patients on weight management, smoking, alcohol consumption and sun safety. Many health professionals (38\%) felt they were not the right person to provide advice and cited lack of resources as a key barrier to advice provision. The majority (95\%) reported being interested in a resource containing relevant lifestyle information that could be given to young people with cancer.

Conclusions: TYA health professionals' awareness of lifestyle guidance and provision of advice regarding health behaviour is sub-optimal. Clear and comprehensive guidance written specifically for TYA health professionals could overcome the reported barriers and improve professionals' confidence in addressing and providing advice on lifestyle to young people with cancer.

\section{Keywords}

Teenage, Young Adult, Cancer Patient, Health Professional, Lifestyle, Advice 
2

\section{Introduction}

Healthy lifestyle choices, such as being physically active and eating a healthy diet, may substantially improve the health and quality of life of teenagers and young adults (TYA) living with and beyond a cancer diagnosis [1-3]. Whereas obesity, smoking, heavy alcohol consumption and negligent sun practices (high levels of sun exposure and poor sun protection) are likely to exacerbate TYA cancer survivors' risk of treatment-related toxicity, chronic disease, secondary cancer and cancer recurrence [4-6]. Given that lifestyle habits formed during adolescence and young adulthood often continue throughout the life-course, it is vital to provide young people who have had a cancer diagnosis are provided with the support necessary to make informed choices about their health behaviour. Health professionals who work with TYA cancer survivors and/or TYA aged childhood cancer survivors are well positioned to provide young people living with and beyond cancer with the right support and guidance to make healthy lifestyle choices $[7,8]$.

Specific lifestyle recommendations written for TYA cancer survivors are contained within the Childrens' Oncology Group (COG) and Scottish Intercollegiate Guidance Network long-term followup guidelines for survivors of childhood, adolescent and young adulthood cancers. Specifically, health professionals are advised to provide health behaviour counselling to young people within the context of late-effects management, especially when regarding the treatment of cardiac problems and metabolic syndrome. While there is currently no formal guidance for TYA cancer survivors receiving treatment, there is general consensus that health professionals caring for young people with cancer have a duty to advocate the importance of healthy lifestyle choices throughout the cancer care pathway [8]. Health behaviour change interventions delivered early within the care pathway have the potential to mitigate the onset of treatment-related comorbidities and prevent the development of unhealthy lifestyle habits, such as inactivity and poor dietary intake, often noted during cancer therapy [9]. Moreover, a recent cross sectional survey of TYA cancer survivors ( $n=216$, mean age $=20$ years) found young people with cancer are receptive to receiving advice and support 
1 on health behaviour from the point of diagnosis onwards [10]. Several organisations (American

2 Cancer Society, CanTeen, Institute of Medicine Livestrong Foundation, National Cancer Institute,

3 National Cancer Comprehensive Network, and Teenagers and Young Adults with Cancer (TYAC)) have

4 produced information resources or position statements relating to lifestyle for the interest of health

5 professionals working with TYA cancer survivors both on and off treatment. Overall, these guidelines and information resources do not differ substantially from those written for adult cancer survivors: all advocate, to varying degrees, the importance of TYA cancer survivors remaining physically active, eating well-balanced diets, limiting alcohol intake, not smoking and being safe in the sun.

9 Existing evidence, although limited to a small number of studies, suggests that many TYA cancer survivors are inactive, have poor dietary quality and engage in risky health behaviours such as drinking, smoking and tanning [11-14]. Nevertheless, previous studies in adult cancer survivors have demonstrated that a health recommendation from a professional can lead to positive lifestyle behaviour change $[15,16]$. However, many TYA cancer survivors report unmet needs in relation to diet and exercise information, and alcohol and drug counselling $[17,18]$. For example data from the United Kingdom (U.K) indicate many young people (36-70\%) had not received advice about their health behaviour since their diagnosis [18]. Discrepancies between the number of TYA cancer survivors reporting a desire to discuss current health behaviours (>50\%) at a follow-up care appointment, and the number reporting actually discussing health behaviour ( $\sim 4 \%$ ) have also previously been found within the U.K [19].

Studies of health professionals working within adult oncology settings suggest that awareness of the importance of lifestyle is generally low and that professionals perceive numerous barriers to providing such advice to their patients [20]. Previous studies of health professionals working with adult cancer survivors have also shown lack of knowledge, lack of time, and patient sensitivity to be perceived barriers to providing lifestyle advice [21-23]. 
1 To our knowledge, little is known about TYA health professionals' familiarity with lifestyle guidance,

2 and their current practices regarding the delivery of lifestyle advice to young people within their

3 care. In addition, there is currently no data on TYA cancer specialists' views of how and when the

4 best time in the cancer care pathway to deliver such advice would be [24]. Therefore, this study

5 aimed to address these gaps in knowledge and explore health professionals working with TYA cancer

6 patients' awareness of lifestyle guidance, level of lifestyle advice provision and views on the format

7 and delivery of lifestyle advice to TYA cancer survivors. Health professionals perceived barriers to

8 providing lifestyle advice were also explored.

\section{Methods}

\section{Participants \& Recruitment}

11 Data were collected via an anonymous online survey. Health professionals (oncologists, 12 haematologists, surgeons, cancer nurses and allied health professionals) working directly with TYA 13 cancer patients in the UK were eligible to complete the survey. From May to November 2015, the web link to the survey was cascaded through the mailing lists of several UK based professional and charitable bodies specialising in the care of TYA cancer patients. The survey was also circulated by existing contacts within the NHS, London Cancer Network and the National Cancer Research Institute Teenage and Young Adult Clinical Studies Group (NRCI TYA CSG). Several reminders about the survey were posted upon the TYAC website and sent to services staff employed or affiliated with CLIC Sargent. TYAC is an organisation within the UK which aims to unite professionals working with young people with cancer and CLIC Sargent is a UK based cancer charity specialising in caring for and supporting young people, and their families, affected by cancer. This study was approved by University College London Ethics Committee (reference 4456/001). By following the online link and completing the survey participants consented to the use of their anonymous data. 
2 The development and design of the survey was based on previous work carried out by Williams and colleagues, who assessed knowledge and predictors of lifestyle advice in health professionals working with adult breast, prostate and colorectal cancer patients [20]. The survey was adapted for use among TYA specialists in consultation with a specialist clinical team led by $\mathrm{RH}$.

6

Demographic questions included those relating to professional speciality and place of work. Professional speciality questions included professional group, length of time in current role, length of time working with young people, best fit description of patient age group, and patient cancer group. Place of work questions investigated hospital affiliation (principal treatment centre/designated hospital/other) and regional affiliation.

Awareness of lifestyle recommendations and guidelines was assessed using the question: 'Are you familiar with any guidelines specifically for TYA cancer patients on any of the following lifestyle topics?' Lifestyle topics included physical activity, diet, weight management, smoking, alcohol consumption and sun safety. TYA health professionals' level of provision of lifestyle advice was determined by two questions: 'With what percentage of your patients do you enquire about the following lifestyle topics?' and 'To what percentage of your patients do you give advice about the following lifestyle topics?' Response options for each questions was 'none', '1-25\%', '26-50\%', '51$75 \%{ }^{\prime},{ }^{\prime}>75 \%$ ' . Health professionals were asked to report barriers or problems they feel would prevent them from providing advice to young people within their care. Response options were based upon barriers previously reported within adult cancer survivorship literature. [21-23]

TYA health professionals' opinions about the format and delivery of lifestyle information to young people with cancer were also explored. Delivery of lifestyle advice was assessed by the question; 'If you were to give advice on the following lifestyle topics, what form does this advice usually take? Response options included 'giving verbal advice', 'giving written advice e.g. leaflet/ pamphlet', 'referring to a website', 'referring to another health professional' or 'other'. Health professionals 
1 were asked to report how best they thought TYA cancer survivors would like to receive information

2 about lifestyle (response options included 'leaflet or pamphlet', 'website', 'mobile app','other') and

3 when in the cancer care pathway ('before treatment', 'during treatment', '0-6 months' 'post-

4 treatment', '6-12 months post treatment', or ' $12+$ months post treatment') lifestyle information

5 should first be introduced to young people with cancer.

\section{Analyses}

7 All survey responses were downloaded and transferred to statistical package SPSS Version 22.

8 Descriptive statistics were produced to describe the demographics of respondents and the 9 proportion of health professionals overall and within each professional group who were familiar with lifestyle guidance, and reported enquiring about health behaviour and providing advice to the majority $(>75 \%)$ of their patients. Descriptive statistics were also produced to report health professionals perceived barriers to providing lifestyle advice and views on the format and delivery of such advice. Exact significance tests for pearsons chi-square analyses were performed to examine potential differences between professional groups regarding familiarity with lifestyle guidance, enquiry about lifestyle and lifestyle advice provision. and coded line-by-line. The process of content analysis as outlined by ELo and Kyngas (2008) was followed. A deductive approach to analysis was taken wherein open responses to each question were grouped together and the content was analysed for patterns or themes. The final coding was analysed and emerging themes were categorised and enumerated. In total, 79 individual open response comments were analysed.

\section{Results}

\section{Response Rate}


1 The exact reach of the survey is unknown as the link to the survey was posted online or cascaded

2 independently via various professional organisations and individuals. 132 health professionals

3 followed the survey link and consented to participating and $114(86 \%)$ of these completed at least

4 one question. Of these $114,95(83 \%)$ completed all of the questions relevant to the current study.

5 There were no significant differences in background demographics between full and partial

6 responders.

\section{$7 \quad$ Sample Characteristics}

8 Sample characteristics are shown in Table 1 and 2. 44\% (n=42) of respondents were nurses. 73\% $9 \quad(n=69)$ identified themselves as working with young people aged $13-24$ years of age and $63 \%(n=60)$ 10 had been working with young people with cancer for more than 5 years. Most respondents (69\%, $\mathrm{n}=66$ ) worked with patients across all cancer sites and were based within a principal treatment centre $(68 \%, n=65)$. The survey had a response from at least one health professional from each of the home nations (England, Scotland, Wales, and Northern Ireland) within the UK, however a large proportion of respondents worked in London $(33 \%, n=31)$ and the South of England $(26 \%, n=25)$.

\section{Awareness of lifestyle guidance}

Table 3 shows the proportion of health professionals who reported being aware of lifestyle guidelines for each health behaviour. More health professionals were aware of guidance relating to physical activity and diet (62\% and $60 \%$ respectively) than weight management, smoking, or alcohol consumption $(40-59 \%) .72 \%(n=68)$ of health professionals were aware of guidance on one or more health behaviour. Of these, $54 \%(n=37)$ could not recall the specific details of the guidance, and most cited charitable bodies such as the Teenage Cancer Trust, Childrens' Cancer and Leukaemia Group 
unaware of any lifestyle guidance for TYA cancer patients. There were no significant differences in the awareness of lifestyle guidance between professional groups.

$4 \quad$ Table 3 shows the proportion of respondents who reported enquiring about lifestyle and providing

5 lifestyle advice to the majority ( $>75 \%$ ) of patients within their care. Overall, approximately half (45-

$655 \%$ ) of professionals surveyed reported enquiring about physical activity, diet, smoking and drinking

7 alcohol with more than three-quarters of their patients. A larger proportion of health professionals

8 asked about and provided advice on diet and physical activity than any of the other health

9 behaviours. Many health professionals surveyed reported that they provide advice on smoking (54\%,

$10 \mathrm{n}=51)$ or alcohol consumption $(48 \%, \mathrm{n}=46)$ to less than a quarter of their patients. Similarly $43 \%$

$11(n=41)$ and $44 \%(n=42)$ of health professionals reported starting conversation with very few $(0-25 \%)$

12 of their patients about weight management and sun safety.

As shown in Table 3, group differences were not observed between physicians and nurses for enquiry or advice provision on any health behaviour. There were no significant differences in the proportion of groups of health professionals who reported enquiring about weight management, or enquiring and providing advice on physical activity. Group differences for enquiry and provision of advice on diet, smoking, alcohol consumption and sun safety were observed between professionals who were physicians/nurses and those who were allied health professionals, or reported their profession as 'other'. There were no significant difference between professional groups who identified themselves working with TYA cancer survivors aged 13-24, versus those who reported working with young people in the older age range (18-39 years) for level of enquiry or provision of advice. 
1 Most $(68 \%-87 \%)$ of respondents reported that very few $(<25 \%)$ of their patients asked them directly

2 for information about weight management, smoking, alcohol consumption or sun safety. Only $27 \%$

$3 \quad(n=28)$ of respondents reported that more than half of their patients ask for information about

4 physical activity: and only $35 \%(n=36)$ reported that more than half of their patients asked for

5 information about diet. The percentage of respondents who reported that no young people in their

6 care asked for specific advice about smoking, drinking and sun safety was $42 \%(n=43), 40 \%(n=41)$,

7 and $36 \%(n=36)$, respectively.

8 Delivery of lifestyle advice

9 As shown in Figure 1, health professionals most commonly opted to provide advice either verbally or 10 by referring their patients to another health professional. Although very few respondents reported 11 actually signposting patients in the direction of information online, open responses suggested electronic resources (such as websites or mobile apps) were considered to be acceptable formats of lifestyle information delivery. In the open response sections of the survey, health professionals reported that the format of information delivery was dependent upon on the needs of the patient and that information should be available in a range of formats to reflect differences in patient preferences. $43 \%$ ( $n=41$ ) of health professionals surveyed believed young people with cancer should first be offered lifestyle advice during treatment, 33\% $(n=31)$ believed lifestyle advice should be first offered before treatment and $22 \%(n=21)$ believed lifestyle advice should be first offered up to 6 months post treatment. Encouragingly, 95\% $(n=91)$ said they would be interested in a resource containing relevant lifestyle information which they could give to their patients, and $76 \%(n=72)$ perceived no problems in providing such information to young people with cancer.

\section{Barriers to providing lifestyle advice}

The most common barrier was 'not being the right person to give advice' $38 \%$ ( $n=36$ ); followed by perception of 'patient feeling too frail or unwell' $36 \%(n=34)$, and perceived lack of patient interest 
$129 \%(n=28) .20 \%(n=19)$ of the health professionals surveyed felt that 'lack of clear guidance' was a

2 barrier to providing lifestyle advice and $17 \%(n=16)$ felt that 'lack of time' was a barrier. No

3

\section{Discussion}

This is the first detailed study to investigate TYA cancer health professionals' knowledge of lifestyle guidance and provision of lifestyle advice to young people within their care. Awareness and familiarity of lifestyle guidance and enquiry about lifestyle behaviours was generally modest among health professionals working with TYA cancer patients, and few TYA health professionals provided lifestyle advice to the majority of patients within their care. The complexity of providing lifestyle advice to young people with cancer, and the need to provide education and tailored resources related to lifestyle was highlighted.

Although the majority (72\%) of survey respondents reported awareness of some lifestyle guidelines, over half (54\%) of these professionals could not recall the source of this guidance, suggesting limited knowledge and familiarity with the guideline content. These findings are similar to studies of health professionals working with adult cancer patients [20]. Low to moderate levels of familiarity with lifestyle guidance among health professionals working with TYA cancer patients is perhaps unsurprising, as although some charitable bodies and medical groups have produced information and guidance about lifestyle topics, these resources often contain a wide array of information on a variety of topics, with only small sections dedicated to lifestyle behaviour and non-specific guidance (such as 'should remain active'). In addition, although guidance relating to lifestyle and cancer has 
1 been produced by bodies such as the World Cancer Research Fund and American Institute for Cancer

2 Research, the focus of this guidance is aimed at adults and contains little specific reference to TYA cancer patients or their needs. The lack of specific lifestyle guidance is likely in part due to a lack of lifestyle intervention trials in the TYA age group and further research in this area is warranted [25, 26].

Less than half of the health professionals surveyed reported giving advice on physical activity or diet to the majority ( $>75 \%)$ of patients in their care. Even fewer (less than a third) reported providing specific advice about weight management, smoking, drinking or sun safety to the majority of their patients. These findings suggest that health professionals working with TYA cancer patients do not routinely initiate conversations about lifestyle behaviours or provide lifestyle advice to their patients. With regard to physical activity, the relatively low level of advice provision may relate to previously debated confusion regarding the safety of encouraging young people with cancer to be active $[27,28]$. This notion could also apply to weight management and diet where 'best practice' recommendations for TYA cancer patients are yet to be devised due to lack of clear evidence. However, for behaviours such as smoking, drinking, and tanning which have well acknowledged negative associations with health [29], the finding that very few health professionals enquire about or provide advice on these topics was surprising. Nevertheless, previous studies have found similar patterns and rates of lifestyle advice provision in surveys of health professionals working with adult cancer patients. $[20,22,30]$.

The low level of enquiry about lifestyle and provision of lifestyle advice from TYA health professionals may in part be explained by professionals' report that very few of their patients ask directly for information about individual lifestyle behaviours. The majority of health professionals surveyed $(68 \%-86 \%)$ reported that less than half of young people within their care asked them directly for lifestyle information. The finding that very few young people with cancer ask directly for information about lifestyle was unexpected given previous reports of high levels of patient interest 
1 in receiving advice on this subject [31,32]. TYA health professionals may perceive low levels of

2 patient enquiry about lifestyle as a lack of interest. However, a recent roundtable workshop

3 addressing the needs and the lifestyle challenges of TYA cancer survivors concluded that health

4 professionals working with TYA cancer patients can and should initiate discussions regarding lifestyle

5 behaviour [8].

6 A large proportion of health professionals selected at least one barrier from the formulated list or

7 cited their own barrier to initiating discussion about or providing advice on lifestyle behaviours.

8 Barriers were most commonly centred on patient-related factors such as lack of interest, being

9 sensitive to their emotional needs and maintaining good patient relationships. Consistent with previous studies of health professionals working in adult oncology [22] one of the most commonly endorsed barriers to providing lifestyle advice was perception of 'not being the right person to provide advice'. This could suggest TYA health professionals believe that addressing lifestyle behaviours and providing lifestyle advice is outside of their professional duty. The need for specifically tailored lifestyle guidance to be made available to all health professionals working with TYA cancer patients was highlighted within open responses. Clear and comprehensive guidance written specifically for TYA health professionals could improve professionals' confidence in addressing and providing advice on lifestyle behaviour whilst remaining sensitive to patient needs and maintaining patient relationships.

Findings from this study suggest that professionals were more likely to address (enquire to patients about) and provide advice on physical activity and diet than weight management, smoking, drinking alcohol, or sun safety. The discrepancies between health behaviours suggest that TYA health professionals may be reluctant to engage in conversation about sensitive issues (such as weight) and 'risky' health behaviours (such as smoking, drinking and unsafe sun practices) and may need additional support and guidance on how best to address these issues, or access evidence-based referral pathways. 
1 Nevertheless, despite the numerous perceived barriers and problems to providing lifestyle advice, over $90 \%$ of health professionals surveyed were interested in a resource containing relevant lifestyle information they could give to their patients. Although the most commonly reported method of advice delivery was verbal, those surveyed acknowledged the need to provide young people with advice in a range of formats, and at a suitable time for the individual patient. The finding that TYA health professionals seldom refer young people within their care to websites may be explained by the current limited availability of lifestyle information specific to young people with cancer. With regard to the timing of delivery, the largest proportion of respondents believed lifestyle advice should first be provided to TYA cancer patients during treatment. This finding is consistent with emerging evidence that healthy lifestyle behaviours during treatment have a positive impact on quality of life and could influence cancer outcomes among young people [33, 34].

Although this is the first study to explore TYA health professionals' knowledge of lifestyle guidance, provision of lifestyle advice and barriers to providing lifestyle advice, a number of limitations must be addressed. Firstly, we do not have data on the proportion of respondents who specifically work in long-term follow up or with specific out-patient groups. In particular, as demonstrated by the wide confidence intervals, the small sample size among some professional groups (specifically physicians) limited our ability to establish precise estimates of between group differences in lifestyle guidance familiarity, enquiry or provision of advice. Moreover, given the broad expertise and clinical roles carried out by allied health professionals it is likely the group differences which were found in the chi-square analyses are arbitrary. However, cancer is relatively uncommon in young people, and the number of health professionals who responded to the survey can be considered as quite large within the TYA health profession. Although the majority of respondents were nurses and were based within London or South East England, survey respondents typically worked with patients with a range of cancer diagnoses and had been working with young people with cancer for more than five years. Health professionals who have been working with TYA cancer patients for longer may have 
1 cancer and therefore may have been more motivated to participate within the survey. This could suggest that, in general, TYA health professionals who did not complete the survey have poorer knowledge about lifestyle and are less likely to give lifestyle advice than those who completed the survey.

The results of this study suggest lifestyle advice provision to young people with cancer may be a complex task. A 'one-size-fits-all' approach to lifestyle information delivery to TYA cancer patients is unlikely to be appropriate given the range of health needs and information comprehension abilities existing within this patient group. Future work should aim to further explore, within a larger sample, TYA health professionals' specific knowledge and understanding of lifestyle guidance and perceived importance of providing lifestyle advice to young people with cancer. The development of clear and succinct guidance on lifestyle for health professionals working with TYA cancer patients is vital as supporting young people with cancer to adopt healthy lifestyle choices which may prevent some of the short-term, and long-term, consequences of cancer treatment experienced by this unique age group [24]. Such information resources should also outline strategies to engage both TYA health professionals and TYA cancer patients in health behaviour promotion initiatives. Such resources have the potential to support professionals to successfully integrate health behaviour promotion into usual care routines. As previously highlighted, the inclusion of specific information about lifestyle and health behaviour within long-term follow-up guidelines for TYA cancer survivors would be a positive first step [35].

In conclusion, this study highlights the complexity of providing lifestyle advice to TYA cancer survivors. Although the majority of respondents thought lifestyle advice should first be provided to TYA cancer patients during treatment a range of barriers and problems to addressing lifestyle behaviour during conversation and providing advice on some specific behaviours were raised. TYA health professionals' high level of interest in receiving resources containing relevant lifestyle information which could be given to patients was encouraging and supports the growing body of 
evidence that health behaviour promotion among TYA cancer survivors is a critical topic to address both in research and clinical practice. Efforts are required to ensure professionals working with TYA cancer survivors have the confidence and skills to address lifestyle during consultations and provide sound advice on individual health behaviours.

Acknowledgements: This work was supported in part by an IMPACT Award co-funded by University College London and CLIC Sargent awarded to Gemma Pugh.

Conflict of Interest: There are no conflicts of interest to report.

\section{References:}

1. Braam, K.I., et al., Physical exercise training interventions for children and young adults during and after treatment for childhood cancer. Cochrane Database Syst Rev, 2013. 4: p. Cd008796.

2. Stolley, M.R., J. Restrepo, and L.K. Sharp, Diet and physical activity in childhood cancer survivors: a review of the literature. Ann Behav Med, 2010. 39(3): p. 232-49.

3. Gilliam, M.B. and D.C. Schwebel, Physical Activity in Child and Adolescent Cancer Survivors: A Review. Health psychology review, 2013. 7(1): p. 92-110.

4. Clarke, S.-A. and C. Eiser, Health behaviours in childhood cancer survivors: A systematic review. European Journal of Cancer, 2007. 43(9): p. 1373-1384.

5. Orgel, E., et al., Impact on survival and toxicity by duration of weight extremes during treatment for pediatric acute lymphoblastic leukemia: A report from the Children's Oncology Group. J Clin Oncol, 2014. 32(13): p. 1331-7.

6. Wilson, C.L., et al., Modifiable Factors Associated With Aging Phenotypes Among Adult Survivors of Childhood Acute Lymphoblastic Leukemia. Journal of Clinical Oncology, 2016. 34(21): p. 2509-2515.

7. Wurz, A. and J. Brunet, Promoting physical activity in adolescent cancer survivors. University of Ottawa Journal of Medicine (UOJM), 2015. 5(1): p. 29.

8. Daniel, C.L., et al., Needs and Lifestyle Challenges of Adolescents and Young Adults with Cancer: Summary of an Institute of Medicine and Livestrong Foundation Workshop Clin J Oncol Nurs, 2015. 19(6): p. 675-81.

9. Barnes, M., et al., Pre-habilitation-Promoting Exercise in Adolescent and Young Adult Cancer Survivors for Improving Lifelong Health - A Narrative Review. Cancer Research Frontiers 2016 2(1): p. 22-32.

10. Pugh, G., et al., The lifestyle information and intervention preferences of teenage and young adult cancer survivors In Press.

11. Hansen, J., et al., Eating Behaviour and BMI in Adolescent Survivors of Brain Tumor and Acute Lymphoblastic Leukemia Journal of Pediatric Oncology Nursing 2014. 31(1): p. 9

12. Zhang, F.F., et al., Comparison of childhood cancer survivors' nutritional intake with US dietary guidelines. Pediatric Blood \& Cancer, 2015. 62(8): p. 1461-1467.

13. Tylavsky, F.A., et al., Nutritional intake of long-term survivors of childhood acute lymphoblastic leukemia: evidence for bone health interventional opportunities. Pediatr Blood Cancer, 2010. 55(7): p. 1362-9. 
14. Marjerrison, S., et al., Smoking, Binge Drinking, and Drug Use Among Childhood Cancer Survivors: A Meta-Analysis. Pediatr Blood Cancer, 2016.

15. Jones, L., et al., Effects of an oncologist's recommendation to exercise on self-reported exercise behavior in newly diagnosed breast cancer survivors: a single-blind, randomized controlled trial. Annals of Behavioral Medicine, 2004. 28(2): p. 105-113.

16. Fisher, A., et al., Recall of physical activity advice was associated with higher levels of physical activity in colorectal cancer patients. BMJ Open, 2015. 5(4).

17. Zebrack, B., Information and service needs for young adult cancer survivors. Supportive Care in Cancer, 2008. 17(4): p. 349-357.

18. Pugh, G., et al., The provision of health behaviour information to teenage and young adult cancer survivors Journal of Adolescent and Young Adult Oncology In Press

19. Michel, G., et al., Follow-up care after childhood cancer: Survivors' expectations and preferences for care. European Journal of Cancer, 2009. 45(9): p. 1616-1623.

20. Williams, K., et al., Health professionals' provision of lifestyle advice in the oncology context in the United Kingdom. European Journal of Cancer Care, 2015. 24(4): p. 522-530.

21. O'Hanlon, É. and N. Kennedy, Exercise in cancer care in Ireland: a survey of oncology nurses and physiotherapists. European Journal of Cancer Care, 2014. 23(5): p. 630-639.

22. Spellman, C., M. Craike, and P.M. Livingston, Knowledge, attitudes and practices of clinicians in promoting physical activity to prostate cancer survivors. Health Education Journal, 2013.

23. Anderson, V.A., et al., Development of executive functions through late childhood and adolescence in an Australian sample. Dev Neuropsychol, 2001. 20(1): p. 385-406.

24. Carretier, J., et al., A Review of Health Behaviors in Childhood and Adolescent Cancer Survivors: Toward Prevention of Second Primary Cancer. Journal of Adolescent and Young Adult Oncology, 2015.

25. Pugh, G., et al., Health Behavior Change Interventions for Teenage and Young Adult Cancer Survivors: A Systematic Review. J Adolesc Young Adult Oncol, 2016. 5(2): p. 91-105.

26. Armenian, S.H., et al., Recommendations for cardiomyopathy surveillance for survivors of childhood cancer: a report from the International Late Effects of Childhood Cancer Guideline Harmonization Group. The Lancet Oncology, 2015. 16(3): p. e123-e136.

27. Spreafico, F., et al., Should we encourage exercise and sports in children and adolescents with cancer? Pediatric Blood \& Cancer, 2014. 61(11): p. 2125-2125.

28. Kumar, M., et al., Sports participation in children and adolescents with immune thrombocytopenia (ITP). Pediatric Blood \& Cancer, 2015: p. n/a-n/a.

29. Klosky, J., et al., Emerging issues in smoking among adolescent and adult cancer survivors. Cancer, 2007. 110(11): p. 2408-2419.

30. Daley, A., et al., What advice are oncologists and surgeons in the United Kingdom giving to breast cancer patients about physical activity? International Journal of Behavioral Nutrition and Physical Activity, 2008. 5(1): p. 46.

31. Murnane, A., et al., Adolescents and young adult cancer survivors: exercise habits, quality of life and physical activity preferences. Supportive Care in Cancer, 2015. 23(2): p. 501-510.

32. Rabin, C., et al., Intervention format and delivery preferences among young adult cancer survivors. Int J Behav Med, 2013. 20(2): p. 304-10.

33. Dubnov-Raz, G., et al., Changes in fitness are associated with changes in body composition and bone health in children after cancer. Acta Paediatrica, 2015. 104(10): p. 1055-1061.

34. Siegel, R., M. Lockhart, and C. Kist, Reducing lifestyle risk in Childhood Cancer Survivors. Acta paediatrica, 2015. 104(10): p. 969-970.

35. Brown, M.C., et al., The views of European clinicians on guidelines for long-term follow-up of childhood cancer survivors. Pediatric Blood \& Cancer, 2015. 62(2): p. 322-328. 
